\title{
Assessment of Individual Fracture Risk: FRAX and Beyond
}

\author{
Joop P. W. van den Bergh • Tineke A. C. M. van Geel • \\ Willem F. Lems • Piet P. Geusens
}

Published online: 22 June 2010

(C) The Author(s) 2010. This article is published with open access at Springerlink.com

\begin{abstract}
The World Health Organization fracture risk assessment tool (FRAX) and the Garvan fracture risk calculator are both widely available tools for individualized fracture risk prediction in daily practice. The FRAX model is implemented in several guidelines and most widely used at present. However, clinicians should take into account the differences between the models, especially with regard to the effect of the number of falls, number and clustering of
\end{abstract}

J. P. W. van den Bergh $(\bowtie)$

Department of Internal Medicine,

VieCuri Medical Centre Noord-Limburg,

P.O. Box 1926, 5900 BX Venlo, The Netherlands

e-mail: jvdbergh@viecuri.nl

\section{J. P. W. van den Bergh}

Faculty of Health Medicine and Life Science,

Department of Internal Medicine, Maastricht University/Nutrim,

P.O. Box 616, 6200 MD Maastricht, The Netherlands

\section{T. A. C. M. van Geel}

Faculty of Health Medicine and Life Science,

Department of General Practice, Maastricht University/Caphri,

P.O. Box 616, 6200 MD Maastricht, The Netherlands

e-mail: t.vangeel@hag.unimaas.nl

\section{W. F. Lems}

Department of Rheumatology, VU Medical Centre,

P.O. Box 7057, 1007 MB Amsterdam, The Netherlands

e-mail: WF.Lems@vumc.nl

\section{P. P. Geusens}

Faculty of Health Medicine and Life Science,

Department of Internal Medicine, Maastricht University/Caphri,

P.O. Box 616, 6200 MD Maastricht, The Netherlands

e-mail: piet.geusens@scarlet.be

\section{P. P. Geusens}

Biomedical Research Center, University Hasselt,

Hasselt, Belgium previous fractures, and the number of clinical risk factors on the outcome of predicted fracture risk. Further development will be needed for optimal integration of bone- and fall-related risks, clustering of fractures, and dosing of risk factors to validate the models in different populations and to validate the ability to select patients who will achieve fracture risk reduction with anti-osteoporosis therapy. FRAX may be used as the primary model, and in patients with recurrent fractures and falls the use of the Garvan model may be of additional value.

Keywords Osteoporosis · Fractures · FRAX · Garvan · Timing of fractures $\cdot$ Risk factors

\section{Introduction}

Osteoporotic fractures are an important cause of morbidity [1] and are linked with significant risk for subsequent fracture and mortality, in both women and men. Epidemiologic studies from North America have estimated the remaining lifetime risk of common fragility fractures in white women 50 years of age to be $17.5 \%$ for hip fracture, $15.6 \%$ for clinically diagnosed vertebral fracture, and $16.0 \%$ for distal forearm fracture. Corresponding risks among men are $6 \%, 5 \%$, and $3 \%$, respectively [2].

Data from the General Practice Research Database in the United Kingdom (UK, which includes $6 \%$ of the UK population) have indicated that the risk is similar in the UK. The lifetime risk of any fracture was found to be $53.2 \%$ at 50 years of age in women, and $20.7 \%$ at the same age in men. Thus, one in two women and one in five men who are 50 years of age will have an osteoporotic fracture in their remaining lifetime [3]. Among women, the 10-year risk of any fracture increased from $9.8 \%$ at 50 years of age to 
$21.7 \%$ at 80 years of age, whereas among men the 10 -year risk remained fairly stable with advancing age with a 10 year risk of any fractures of $7.1 \%$ at 50 years to $8.0 \%$ at 80 years.[3] The health burden of osteoporotic fractures is likely to rise, which is partly due to an increased life expectancy and to changes in lifestyle (eg, less exercises/ mobility, less calcium intake). Therefore, understanding the epidemiology of this disease is essential in trying to develop strategies to target individuals at high risk for fracture.

\section{Assessment of Fracture Risk}

\section{Bone Mineral Density Measurement}

In 1994, the World Health Organization published diagnostic criteria for osteoporosis defined in terms of bone mineral density (BMD) measurement with dual-energy X-ray absorptiometry as a T-score $\leq-2.5$ standard deviation (SD) below the young mean [4]. Recently, it was proposed that the reference standard should be based on BMD measurement made at the femoral neck because this site has been the most extensively validated, and provides a gradient of fracture risk as high as or higher than that of many other techniques [5].

In prospective and cross-sectional epidemiologic studies it has been shown that there is an inverse relationship between bone mass and fracture. The risk of osteoporotic fracture increases continuously as BMD declines, resulting in a 1.5- to threefold increase in risk of fracture for each SD decrease in BMD [6]. Advanced age and low BMD are strongly associated with higher fracture risk in postmenopausal women [7], and data from multiple randomized controlled trials provide evidence for fracture prevention in individual patients with osteoporosis [8]. Up to now, many guidelines recommend the assessment of BMD in patients with clinical risk factors (CRFs) as a selection for whom to treat. Using T-scores has many benefits, because T-scores are simple and widely used, have a good correlation with fracture risk, and can detect some high-risk patients. However, the majority of fractures occur in the large group of older women without osteoporosis, but with BMD in the osteopenic range [9]. Furthermore, several other independent risk factors for fractures, over and above that reflected by BMD, have been identified [10, 11]. Thus, BMD will not reliably predict all individuals who will sustain a fracture from those who will not.

Assessment of High-Risk Patients

\section{The Fracture Risk Assessment Tool}

Given the increasing evidence now suggesting that T-scores alone are not optimal predictors of fracture risk, the World
Health Organization Metabolic Bone Disease Group recently developed other ways to assess fracture risk. In 2008 the fracture risk assessment tool (FRAX) was released using CRFs with and without BMD for fracture risk predication in men and women $[1,12]$. The development of the FRAX tool has been supported by organizations including the International Osteoporosis Foundation, National Osteoporosis Foundation (NOF), the American Society for Bone and Mineral Research, and the International Society for Clinical Densitometry. The FRAX tool (http://www.shef.ac.uk/ FRAX) computes the 10-year probability of hip fracture or a major osteoporotic fracture. A major osteoporotic fracture is defined as a clinical spine, hip, forearm, and humerus fracture. An estimation of the 10-year fracture probability is calculated in men or women using age, body mass index, and CRFs (Table 1) [13-22]. The 10-year risk of fractures can be calculated with or without femoral neck BMD in the model.

Probability models need to be calibrated to the epidemiology of fracture and death for any particular region or country, because of considerable variations in fracture probability and mortality in different regions of the world [7, 23]. Therefore, FRAX models have been developed from study cohorts from Europe, North America, Asia, and Australia. Additionally, in a Poisson regression model, mortality is taken into account as a competing risk [24]. In the absence of a FRAX model for a particular country, a surrogate country should be chosen, based on the likelihood that it is representative of the index country.

In recently updated guidelines, FRAX is included as a tool for case finding for identifying postmenopausal women at high risk for fractures, for selecting subjects who would need a BMD measurement, and for treatment decisions (NOF) in the United States [25] and (National Osteoporosis Guideline Group [NOGG]), in collaboration with many Societies) in the UK $[26,27]$. It is expected that FRAX will also be helpful in designing fracture prevention studies and in reimbursement issues, as patients at increased probability of fracture can be identified beyond currently accepted reimbursement thresholds for BMD [28].

In spite of its strengths, FRAX also has limitations. First, the model has not been validated in randomized clinical intervention trials focusing on the prevention of fractures in patients who are included based on FRAX data that are available. In post hoc analyses of intervention studies with clodronate and bazedoxifene, the estimation of an individual's 10-year probability of fracture by the FRAX algorithm identified patients at high risk for fracture who will respond to antiresorptive therapy, irrespective of BMD results [29, $30,31 \cdot 32]$. However, once femoral neck BMD and age are known, the eight additional risk factors in FRAX did not significantly improve the prediction of vertebral fracture in post hoc analyses of the alendronate studies. A combination 
Table 1 Comparison of risk factors and predicted fracture risk between the FRAX and Garvan fracture risk calculator

\begin{tabular}{|c|c|}
\hline FRAX tool & Garvan nomogram \\
\hline Risk factors & Risk factors \\
\hline Age & Age \\
\hline Sex & Sex \\
\hline Body weight & Body weight $\mathrm{t}^{\mathrm{a}}$ \\
\hline Femoral neck BMD & Femoral neck BMD ${ }^{\mathrm{a}}$ \\
\hline History of prior fractures ${ }^{\mathrm{b}}$ & $\begin{array}{l}\text { History of prior fractures } \\
\text { after age } 50 \mathrm{y}^{\mathrm{c}}\end{array}$ \\
\hline $\begin{array}{l}\text { Height } \\
\text { Parent with hip fracture }\end{array}$ & \multirow[t]{6}{*}{$\begin{array}{l}\text { History of falls in the } \\
\text { previous } 12 \mathrm{mo}\end{array}$} \\
\hline Current smoking & \\
\hline $\begin{array}{l}\text { Glucocorticoid exposure } \\
>3 \mathrm{mo} \geq 5 \mathrm{mg} / \mathrm{d}\end{array}$ & \\
\hline Rheumatoid arthritis & \\
\hline Secondary osteoporosis ${ }^{\mathrm{d}}$ & \\
\hline Alcohol $\geq 3$ units $/ \mathrm{d}$ & \\
\hline $\begin{array}{r}\text { Predicted fractures } \\
\text { (10-y probability) }\end{array}$ & $\begin{array}{l}\text { Predicted fractures (5- and } \\
10-y \text { probability) }\end{array}$ \\
\hline Hip & Hip \\
\hline Spine & Clinical spine \\
\hline Wrist & Wrist \\
\hline \multirow[t]{9}{*}{ Humerus } & Humerus \\
\hline & Pelvis \\
\hline & Rib \\
\hline & Sternum \\
\hline & Distal femur \\
\hline & Proximal tibia/fibula \\
\hline & Distal tibia/fibula \\
\hline & Patella \\
\hline & Hands and feet (not digits) \\
\hline
\end{tabular}

$B M D$ bone mineral density, FRAX fracture risk assessment tool

${ }^{a}$ Either body weight or BMD is used in the Garvan nomogram.

${ }^{\mathrm{b}}$ A previous fracture in adult life occurring spontaneously, or a fracture arising from trauma that, in a healthy individual, would not have resulted in a fracture.

${ }^{\mathrm{c}}$ Excluding major fractures.

${ }^{\mathrm{d}}$ These include untreated hypogonadism in men and women (eg, premature menopause, bilateral oophorectomy or orchidectomy), anorexia nervosa, chemotherapy for breast cancer, hypopituitarism, inflammatory bowel disease (eg, Crohn's disease and ulcerative colitis), prolonged immobility (eg, spinal cord injury, Parkinson's disease, stroke, muscular dystrophy), ankylosing spondylitis, organ transplantation, type 1 diabetes, thyroid disorders (eg, untreated hyperthyroidism, overtreated hypothyroidism), chronic obstructive pulmonary disease, osteogenesis imperfecta in adults, chronic malnutrition, or malabsorption and chronic liver disease

of baseline radiographic vertebral fracture, femoral neck $\mathrm{BMD}$, and age was found to be the strongest predictor of future vertebral fracture [33]. Further studies will be needed to validate the efficacy of treatment in terms of fracture risk reduction when subjects at high risk for fractures are being treated based on FRAX in the absence of a morphometric vertebral fracture, hip fracture, or a low BMD, which is the case in most patients presenting with a nonvertebral fracture. Second, FRAX can only be used in untreated patients. Third, the increased subsequent fracture risk after an initial fracture is considered constant over time in FRAX, so the calculation by FRAX over a 10-year period does not take into account the clustering in time of subsequent fracture risk after an initial fracture. However, subsequent fracture risk fluctuates over time and is highest within the first years after an initial fracture, as has been shown for repeat vertebral, nonvertebral, and hip fractures [34-36, 37•]. Fourth, fall-related risks were explicitly excluded from the FRAX calculations. Reasons were the lack of standardized evaluation methods and the lack of fracture prevention data with fall prevention measures, which decrease the risk of falls (FRAX). However, fall risks were recognized as a risk for fractures independently of bone-related risks, especially for nonvertebral fractures, including hip fractures [8, 38, 39]. Kayan et al. [32] reported that fall risk does not significantly impact on the antifracture efficacy of clodronate, suggesting that after confirmation with other agents, fall risk may be incorporated into risk assessment tools designed to target skeletal therapies. Given this observation, it would be an additional value to take fall risk into account in the assessment of fracture risk. Finally, FRAX does not include several additional risk factors for fractures, such as the number of causes of secondary osteoporosis, the dose and duration of glucocorticoid use [40], characteristics of previous fractures (location, number, and severity) [41], and vitamin D deficiency [42].

\section{The Garvan Fracture Risk Calculator}

In 2007, another prognostic nomogram for individualizing the risk of hip fracture was developed based on the Dubbo Osteoporosis Epidemiology Study data [43]. In a subsequent study, the model was extended for prediction of the 5- and 10-year risk of any fragility fracture [44•]. The model uses CRFs such as history of prior fracture $(1,2$, or more than 2), history of fall during the past 12 months $(1,2$, or more than 2), age, and BMD (Table 1). The authors developed two models, one with weight and one with BMD. The difference in predictive accuracy between the model with BMD and the model with weight is only modest, and therefore, if BMD is not available, the model with weight can be used in clinical practice [44]. The Garvan tool takes into account the history and number of recent falls $(1,2$, and $>2)$ and the number of previous fractures $(1,2$, and $>2)$. It also predicts more types of fractures than FRAX. The Garvan tool allows 5- and 10year fracture calculations, whereas the FRAX calculates the 10-year fracture risk [44]. A limitation of the Garvan tool is 
that it does not include other risk factors as in FRAX, and therefore, might underestimate fracture risk when many CRFs are present. Another limitation is its availability only in subjects older than 60 years of age and that it is based on an Australian population only, in contrast to FRAX.

When comparing the FRAX and Garvan tool on a theoretic basis, the outcome of the fracture probability of both tools differs depending upon the risk profile of patients. In patients without CRFs, the fracture risk calculation is higher with the Garvan tool than with FRAX, since the number of fractures predicted by Garvan is higher than by FRAX [45]. Also, when using the Garvan tool, calculated fracture risk increases according to the number of previous fractures after 50 years of age and the number of falls in the last 12 months, in contrast to FRAX (Fig. 1). Conversely, calculated fracture risk increases with the number of CRFs with FRAX, whereas Garvan does not take additional CRFs into account (Fig. 2) [45]. In a recent Australian cohort analysis, both approaches were reasonably accurate in women, but FRAX discriminated fracture risk poorly in men [46]. However, it there is no available Australian database for FRAX, and UK and US databases were used instead in this study. In another recent study, in which a comparison is made between both models in a population of 2,012 Polish women, the mean conformity for any fracture risk was $79.1 \%$ and for hip fracture $79.5 \%$; however, it appeared that for prediction of hip fractures, the cumulative role of falls and multiple previous fractures was stronger than other CRFs [38].

\section{The Use of FRAX in Guidelines}

Until recently, the majority of clinical guidelines for the management of osteoporosis have made recommendations for intervention based on BMD T-scores [1]. In 2008 the NOGG in collaboration with many Societies in the UK recommended an approach for decision making based on fracture probabilities derived from FRAX that can be applied to men and women. The NOGG provided intervention thresholds (the fracture probability at which intervention is recommended) and assessment thresholds (the fracture probabilities at which a BMD test might or might not be recommended). Probabilities of a major osteoporotic and hip fracture can be plotted at the NOGG website (http://www.shef.ac.uk/NOGG) available through FRAX, showing whether the patients should be reassured, need a BMD measurement, or can be considered for treatment. The intervention threshold at each age is set at a risk equivalent to that associated with a prior fracture and therefore rises with age [27]. Men and women with 10-year major fracture probabilities below the lower assessment threshold can be reassured. Men and women with probabilities above the upper assessment threshold can be considered for treatment. Men and women with probabilities between the upper and lower assessment threshold should be referred for BMD measurements and their fracture probability reassessed.

The NOF recommends using FRAX when the decision to treat or not to treat is uncertain. It is primarily intended for postmenopausal women and men 50 years of age and older who have T-scores between $-1.0 \mathrm{SD}$ and $-2.5 \mathrm{SD}$ and who are not on treatment, and who have not had spine or hip fractures. The guideline recommends drug treatment if the FRAX 10 -year probability exceeds $20 \%$ for four major fractures or $3 \%$ risk for hip fracture based on economic models suggesting cost-effectiveness of osteoporosis treatment above these percentages [25, 47, 48].

The use of FRAX as intended in both guidelines changes current clinical management and may be difficult to
Fig. 1 Ten-year calculated risk of osteoporotic fractures according to the fracture risk assessment tool (FRAX) (UK) and Garvan tool based on the number of recent falls (the last 12 months) and previous fractures after 50 years of age (women, 60 years, $70 \mathrm{~kg}$, $170 \mathrm{~cm}$, no bone mineral density measurement)

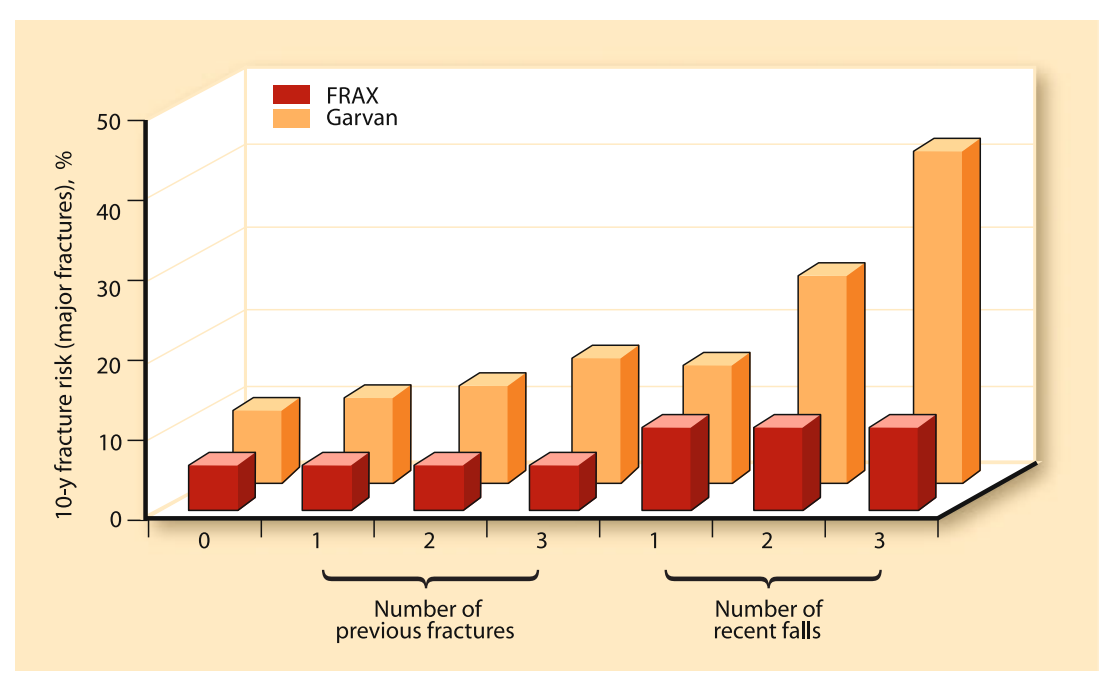


Fig. 2 Ten-year calculated risk of osteoporotic fractures according to the fracture risk assessment tool (FRAX) (UK) and Garvan tool based on the number of clinical risk factors (women, 60 years, $60 \mathrm{~kg}$, $170 \mathrm{~cm}$, T-score femoral neck $=-2.5$ )

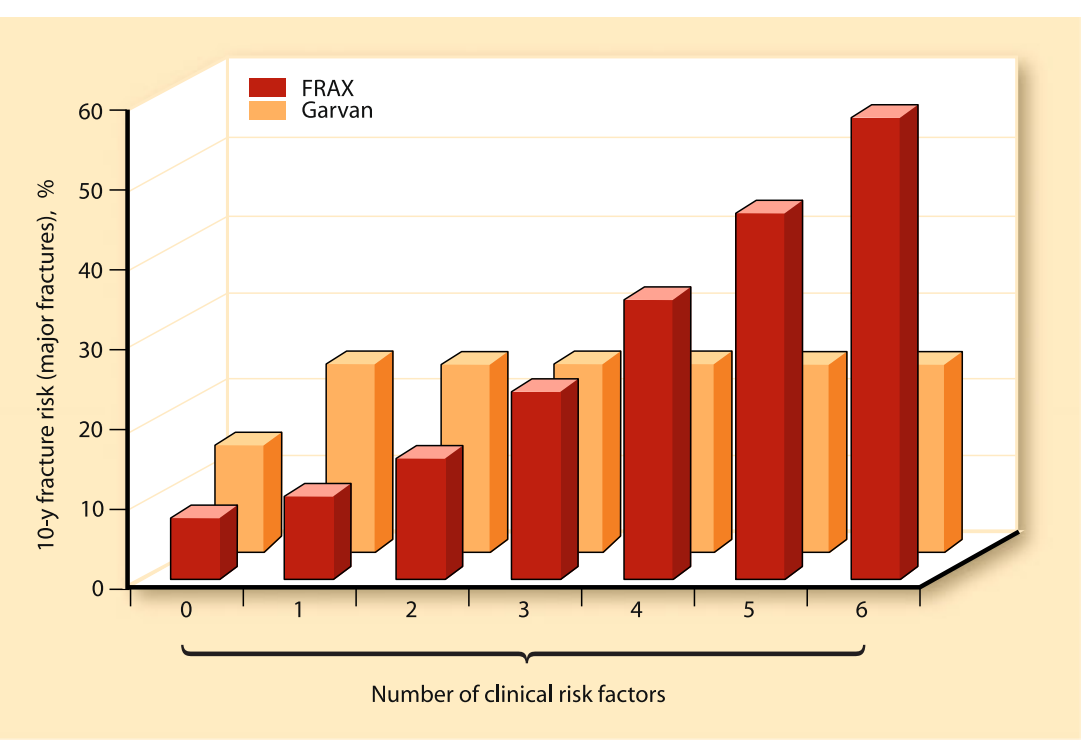

interpret for clinicians. For example, there are patients with one CRF who should be referred for a BMD measurement according to the NOGG guide, and when having osteoporosis (T-score $\leq-2.5$ ) should not be treated according to the reassessed FRAX probability because it is too low to justify treatment according to the NOGG. In contrast, according to NOF guidelines, patients with osteopenia who have a 10 year major fracture risk greater than $20 \%$ based on the presence of CRFs would be treated despite the fact that they do not have osteoporosis.

Additional interpretation issues may arise because a discrepancy may exist between BMD values at the lumbar spine and femoral neck, especially among women around 50 years of age who may have low lumbar spine BMD with normal femoral neck BMD. It is anticipated that FRAX will be incorporated into bone density software in the United States in the near future, so that BMD results will be combined with the FRAX 10-year fracture probability. Patients and physicians will be routinely provided with information on fracture risk that adds to that derived from BMD alone [49]. Physicians should be aware of the fact that BMD is a risk factor for fracture, and the association between BMD and risk is a continuous one despite the Tscore cutoff of $-2.5 \mathrm{SD}$ that is used for the clinical diagnosis of osteoporosis [50]. Conversely, they also need to be educated about the strengths and limitations of FRAX and how calculated risks can be used in clinical practice for individual patients. Whether to add FRAX to a BMD result in the report to the physician is thus still a matter of debate [39, 49].

The FRAX tool may be inappropriate in a number of situations, most notably when osteoporosis treatment is clearly needed (eg, in the presence of a vertebral fracture), but may be very useful for convincing patients who have low 10-year fracture risks that osteoporosis treatment is unnecessary even when the BMD values are in the lower range and to show patients that fracture risk increases with the occurrence of additional risk factors (eg, smoking, recent insufficiency fracture, or glucocorticoid therapy) regardless of BMD. In patients with high fall risk, a recent previous fracture, and/or multiple previous fractures, the 10-year fracture risks may be underestimated by FRAX, as shown with the use of the Garvan algorithm in such cases.

\section{Conclusions}

In daily practice, there is an urgent need for clinicians to have a model that estimates the absolute fracture risk in their patients, since decisions about whether or not to treat patients with anti-osteoporotic drugs should be based on the efficacy of these drugs, but also on the fracture risk. The FRAX and the Garvan fracture risk calculator are both widely available tools for individualized fracture risk prediction in daily practice. The FRAX model is implemented in the NOF, National Osteoporosis Society, and NOGG guidelines and most widely used at present. Its advantage is that it is easy to use in clinical practice for selecting patients at high risk for fractures, thus it is useful for clinical decision making, case finding and is a valuable tool for patient education. Clinicians should take into account the differences between the models especially with respect to the effect of the number of falls and fractures on fracture risk that is implemented in the Garvan model but not in FRAX and the effect of the number of CRFs on fracture risk in the FRAX, not in the Garvan model; they also need to be aware of possible limitations of the models when using them for individual patient management. Both 
models still need to be validated in different populations before they can be generalized to other populations and further studies will be needed to validate their contribution in selecting patients who will achieve fracture risk reduction with anti-osteoporosis therapy. Further studies will be needed to develop a case-finding algorithm that integrates bone- and fall-related risks, the clustering of fractures, and the dosing of risk factors (eg, number and severity of previous fractures, dose of glucocorticoids, timing, and number of falls). With the current available algorithms, a possible clinical application may be to use FRAX as the primary model and to consider using Garvan in patients with recurrent fractures and falls.

Disclosure No potential conflicts of interest relevant to this article were reported.

Open Access This article is distributed under the terms of the Creative Commons Attribution Noncommercial License which permits any noncommercial use, distribution, and reproduction in any medium, provided the original author(s) and source are credited.

\section{References}

Papers of particular interest, published recently, have been highlighted as:

- Of importance

1. Kanis JA, on behalf of the World Health Organization Scientific Group: Assessment of Osteoporosis at the Primary Health-Care Level. Technical report. University of Sheffield, UK: WHO Collaborating Centre; 2008.

2. Holroyd C, Cooper C, Dennison E: Epidemiology of osteoporosis. Best Pract Res Clin Endocrinol Metab 2008, 22:671-685.

3. van Staa TP, Dennison EM, Leufkens HG, Cooper C: Epidemiology of fractures in England and Wales. Bone 2001, 29:517-522.

4. Assessment of fracture risk and its application to screening for postmenopausal osteoporosis. Report of a WHO Study Group [no authors listed]. World Health Organ Tech Rep Ser 1994, 843:1129.

5. Kanis JA, McCloskey EV, Johansson H, et al.: A reference standard for the description of osteoporosis. Bone 2008, 42:467475 .

6. Marshall D, Johnell O, Wedel H: Meta-analysis of how well measures of bone mineral density predict occurrence of osteoporotic fractures. BMJ 1996, 312:1254-1259.

7. Cummings SR, Melton LJ: Epidemiology and outcomes of osteoporotic fractures. Lancet 2002, 359:1761-1767.

8. Geusens P: Strategies for treatment to prevent fragility fractures in postmenopausal women. Best Pract Res Clin Rheumatol 2009, 23:727-740.

9. Siris ES, Chen YT, Abbott TA, et al.: Bone mineral density thresholds for pharmacological intervention to prevent fractures. Arch Intern Med 2004, 164:1108-1112.

10. Kanis JA: Diagnosis of osteoporosis and assessment of fracture risk. Lancet 2002, 359:1929-1936.
11. Cauley JA, Wu L, Wampler NS, et al.: Clinical risk factors for fractures in multi-ethnic women: the Women's Health Initiative. J Bone Miner Res 2007, 22:1816-1826.

12. Kanis JA, Johnell O, Oden A, et al.: FRAX and the assessment of fracture probability in men and women from the UK. Osteoporos Int 2008, 19:385-397.

13. Kanis JA, Oden A, Johnell O, et al.: The use of clinical risk factors enhances the performance of BMD in the prediction of hip and osteoporotic fractures in men and women. Osteoporos Int 2007, 18:1033-1046.

14. Kanis JA, Johansson H, Oden A, et al.: A meta-analysis of milk intake and fracture risk: low utility for case finding. Osteoporos Int 2005, 16:799-804.

15. De Laet C, Kanis JA, Oden A, et al.: Body mass index as a predictor of fracture risk: a meta-analysis. Osteoporos Int 2005, 16:1330-1338.

16. Kanis JA, Borgstrom F, De Laet C, et al.: Assessment of fracture risk. Osteoporos Int 2005, 16:581-589.

17. Kanis JA, Johansson H, Johnell O, et al.: Alcohol intake as a risk factor for fracture. Osteoporos Int 2005, 16:737-742.

18. Kanis JA, Johnell O, Oden A, et al.: Smoking and fracture risk: a meta-analysis. Osteoporos Int 2005, 16:155-162.

19. Johnell O, Kanis JA, Oden A, et al.: Predictive value of BMD for hip and other fractures. J Bone Miner Res 2005, 20:1185-1194.

20. Kanis JA, Johansson H, Oden A, et al.: A family history of fracture and fracture risk: a meta-analysis. Bone 2004, 35:1029-1037.

21. Kanis JA, Johnell O, De Laet C, et al.: A meta-analysis of previous fracture and subsequent fracture risk. Bone 2004, 35:375-382.

22. Kanis JA, Johansson H, Oden A, et al.: A meta-analysis of prior corticosteroid use and fracture risk. J Bone Miner Res 2004, 19:893-899.

23. Kanis JA, Johnell $\mathrm{O}$, De Laet $\mathrm{C}$, et al.: International variations in hip fracture probabilities: implications for risk assessment. J Bone Miner Res 2002, 17:1237-1244.

24. Oden A, Dawson A, Dere W, et al.: Lifetime risk of hip fractures is underestimated. Osteoporos Int 1998, 8:599-603.

25. National Osteoporosis Foundation: Clinician's Guide to Prevention and Treatment of Osteoporosis. Washington DC: National Osteoporosis Foundation; 2008.

26. Kanis JA, McCloskey EV, Johansson $\mathrm{H}$, et al.: Case finding for the management of osteoporosis with FRAX - assessment and intervention thresholds for the UK. Osteoporos Int 2008, 19:1395-1408.

27. Compston J, Cooper A, Cooper C, et al.: Guidelines for the diagnosis and management of osteoporosis in postmenopausal women and men from the age of 50 years in the UK. Maturitas 2009, 62:105-108.

28. Lippuner K, Johansson H, Kanis JA, Rizzoli R: FRAX assessment of osteoporotic fracture probability in Switzerland. Osteoporos Int 2010, 21:381-389.

29. Kanis JA, Johansson H, Oden A, McCloskey EV: Bazedoxifene reduces vertebral and clinical fractures in postmenopausal women at high risk assessed with FRAX. Bone 2009, 44:1049-1054

30. Kanis JA, Oden A, Johansson H, et al.: FRAX and its applications to clinical practice. Bone 2009, 44:734-743.

31. - McCloskey EV, Johansson H, Oden A, et al.: Ten-year fracture probability identifies women who will benefit from clodronate therapy-additional results from a double-blind, placebocontrolled randomised study. Osteoporos Int 2009, 20:811-817. This study demonstrates that the estimation of an individual's 10year probability of fracture by the FRAX algorithm identifies patients at high risk for fracture who will respond to bisphosphonate therapy. 
32. Kayan K, Johansson H, Oden A, et al.: Can fall risk be incorporated into fracture risk assessment algorithms: a pilot study of responsiveness to clodronate. Osteoporos Int 2009, 20:2055-2061.

33. Donaldson MG, Palermo L, Schousboe JT, et al.: FRAX and risk of vertebral fractures: the fracture intervention trial. J Bone Miner Res 2009, 24:1793-1799.

34. Lindsay R, Silverman SL, Cooper C, et al.: Risk of new vertebral fracture in the year following a fracture. JAMA 2001, 285:320-323.

35. Center JR, Bliuc D, Nguyen TV, Eisman JA: Risk of subsequent fracture after low-trauma fracture in men and women. JAMA 2007, 297:387-394.

36. Ryg J, Rejnmark L, Overgaard S, et al.: Hip fracture patients at risk of second hip fracture: a nationwide population-based cohort study of 169,145 cases during 1977-2001. J Bone Miner Res 2009, 24:1299-1307.

37. - van Geel TA, van Helden S, Geusens PP, et al.: Clinical subsequent fractures cluster in time after first fractures. Ann Rheum Dis 2009, 68:99-102. This population-based study demonstrates that from menopause onward, clinical fractures cluster in time, indicating the need for early action to prevent subsequent fractures.

38. Pluskiewicz W, Adamczyk P, Franek E, et al.: Ten-year probability of osteoporotic fracture in 2012 Polish women assessed by FRAX and nomogram by Nguyen et al.-Conformity between methods and their clinical utility. Bone 2010, 46:1661-1667.

39. Siris ES, Baim S, Nattiv A: Primary care use of FRAX: absolute fracture risk assessment in postmenopausal women and older men. Postgrad Med, 122:82-90.

40. van Staa TP, Geusens P, Kanis JA, et al.: A simple clinical score for estimating the long-term risk of fracture in post-menopausal women. QJM 2006, 99:673-682.
41. Saag KG, Geusens P: Progress in osteoporosis and fracture prevention: focus on postmenopausal women. Arthritis Res Ther 2009, 11:251.

42. Cauley JA, Lacroix AZ, Wu L, et al.: Serum 25-hydroxyvitamin D concentrations and risk for hip fractures. Ann Intern Med 2008, 149:242-250.

43. Nguyen ND, Frost SA, Center JR, et al.: Development of a nomogram for individualizing hip fracture risk in men and women. Osteoporos Int 2007, 18:1109-1117.

44. - Nguyen ND, Frost SA, Center JR, et al.: Development of prognostic nomograms for individualizing 5-year and 10-year fracture risks. Osteoporos Int 2008, 19:1431-1444. This article describes the development of the Garvan nomogram for individualized 5-year and 10-year fracture risk prediction.

45. van Geel TA, van den Bergh JP, Dinant GJ, Geusens PP: Individualizing fracture risk prediction. Maturitas, 65:143-148.

46. Sandhu SK, Nguyen ND, Center JR, et al.: Prognosis of fracture: evaluation of predictive accuracy of the FRAX algorithm and Garvan nomogram. Osteoporos Int 2010, 21:863-871.

47. Donaldson MG, Cawthon PM, Lui LY, et al.: Estimates of the proportion of older white women who would be recommended for pharmacologic treatment by the new U.S. National Osteoporosis Foundation Guidelines. J Bone Miner Res 2009, 24:675-680.

48. Dawson-Hughes B, Tosteson AN, Melton LJ 3rd, et al.: Implications of absolute fracture risk assessment for osteoporosis practice guidelines in the USA. Osteoporos Int 2008, $19: 449-458$

49. Watts NB, Siris ES, Cummings SR, Bauer DC: Filtering FRAX. Osteoporos Int 2010, 21:537-541.

50. McCloskey E, Compston J, Cooper C: The US FRAX filter: avoiding confusion or hindering progress? Osteoporos Int 2010, 21:885. 